\title{
Stabilization of switched linear systems using differential evolution algorithm
}

\author{
Houssem HOSNI ${ }^{1}$, Walid BEN MABROUK ${ }^{2}$, Noureddine LIOUANE ${ }^{1}$ \\ ${ }^{1}$ Ecole Nationale d'Ingénieurs de Monastir, Rue Ibn El Jazzar, Monastir 5019, Tunisia \\ ${ }^{2}$ Institut supérieur des sciences appliquées et de Technologie de Gafsa, \\ Sidi Ahmed Zarrouk, Gafsa, 2112, BP 116. Tunisia
}

\begin{abstract}
This paper describes the development of a new methodology for searching a common quadratic Lyapunov function for switched linear systems based on differential evolution algorithm. This method aims to assure the stabilization of this class of systems. To illustrate the proposed methodology two examples are exploited.
\end{abstract}

Keywords: Common quadratic Lyapunov function; differential evolution algorithm; switched linear systems; stabilization.

\section{Introduction}

During the past several years, there have been many researches activities about the stability analysis and the stabilization for switched systems, considering that the switched systems have numerous applications in the control of mechanical systems, process control, automotive industry, power systems, aircraft and traffic control, and many other fields. [1][2][3].

The switched system is a class of hybrid dynamical systems . A switched system consists of a family of a finite number of subsystems which are described by differential or difference equations and a switching signal that a logical law indicates an active subsystem.

It is important to be able to ensure the existence of a common quadratic Lyapunov function (CQLF) for a given switched system because this is proof that the system is asymptotically stable. There exists a switched quadratic Lyapunov function to check asymptotic stability of the switched discrete-time system in [4].

In recent years, several researches have been done to analyze the stability of switched systems. Which may be mentioned as those based on inverse Lyapunov theorem, switched quadratic Lyapunov functions and common quadratic Lyapunov functions (CQLFs) [5].

In addition, the existence of a CQLF has been widely explored, by directing the efforts to the determination of conditions of existence and non-existence of a CQLF [5][6]. The design of a method for finding a CQLF given its existence by solving the linear matrix inequalities (LMI).

A concise description of stability analysis and stabilization of the switched discrete-time systems using Lyapunov stability theorem and genetic algorithm has been recently developed [7]. The development of a new methodology for calculating a common quadratic Lyapunov function CQLF based on particle swarm optimization (PSO) once the existence of a CQLF has been assured [8]. Moreover, a method for determining the non-existence of a common quadratic Lyapunov function for switched linear systems based on particle swarm optimization was designed and was described [9]. Besides, other methods for finding a CQLF was developed with the aim of improving the limitations of LMI tools, and the most outstanding is the gradient-based method [10].

As a direct consequence of analyzing the scenario described above, it is clear that a new methodology for the calculation of a CQLF and stabilization of switched linear systems, more efficient and less restrictive, must be developed. Then in this article, a new general methodology based on the differential evolution algorithm technique is proposed. The performance of the proposed method is validated through an example of application. This paper is organized as follows: The differential evolution algorithm is described in Section 2. In section 3, the stabilization of switched system is developed. Section 4 is devoted to presenting two examples of application for switched systems in order to illustrate our result, and a concluding section is presented in Section 5.

\section{DIFFERENTIAL EVOLUTION ALGORITHM A. Differential evolution algorithm}

Differential evolution (DE) belongs to to the family of metaheuristics. It was invented by Storn and Price in 1995 [11], [12]. The majority of search results on differential evolution algorithms have not given an important number of theoretical results [12], [13], [14], [15]. The majority of the results are eventually focused on the operations of the mutation, crossover and selection that are related to objective function values and characteristics.

DE algorithm was inspired from the phenomena of nature as PSO and genetic algorithm. It is a simple and powerful algorithm that simulates natural evolution combined with relations of mutation and crossover to multiple search directions based on the distribution of generate solutions in the current population. This algorithm is also used for system optimization. Now, in this paper we use it to stabilize the switched systems. Each matrix $P_{i, G}$ in the population at generation $G$, called at this moment of reproduction as the target matrix will generate one offspring $P_{i, G+1}$, called trial matrix. The trial matrix is generated with the following process: A search direction is defined by calculating the 
difference between a pair of matrices $P_{r 1, G}$ and $P_{r 2, G}$, called differential matrix, both of them are chosen at random from the population. This difference matrix is also scaled by using a user-defined parameter $\alpha \geq 0$. This scaled difference matrix is then added to a third matrix $P_{r 3, G}$, called base matrix. As a result, a new matrix $P_{i, G+1}$ is obtained, known as the mutation matrix, according to the following equation of evolution [16] $P_{i, G+1}=P_{r 3, G}+\alpha\left(P_{r 1, G}-P_{r 2, G}\right)$

After that, this mutation matrix is recombined with the target matrix (also called parent matrix) to generate a trial matrix (child matrix) by using discrete recombination (usually binomial crossover) controlled by a crossover parameter $0 \leq C R \leq 1$ whose value determines how similar the trial matrix will be with respect to the target matrix. In this paper, the following crossover operations are used:

$$
P(i, j)=\left\{\begin{array}{lr}
P_{i, G} & \text { if } \operatorname{rand}[0,1]<C r \\
P_{i, G+1} & \text { otherwise }
\end{array}\right.
$$

with $i$ is matrix number and $G$ is generation number and rand $[0,1]$ is randomly chosen from $[0,1]$.

The differential evolution algorithm could be summarized in the following flowchart:

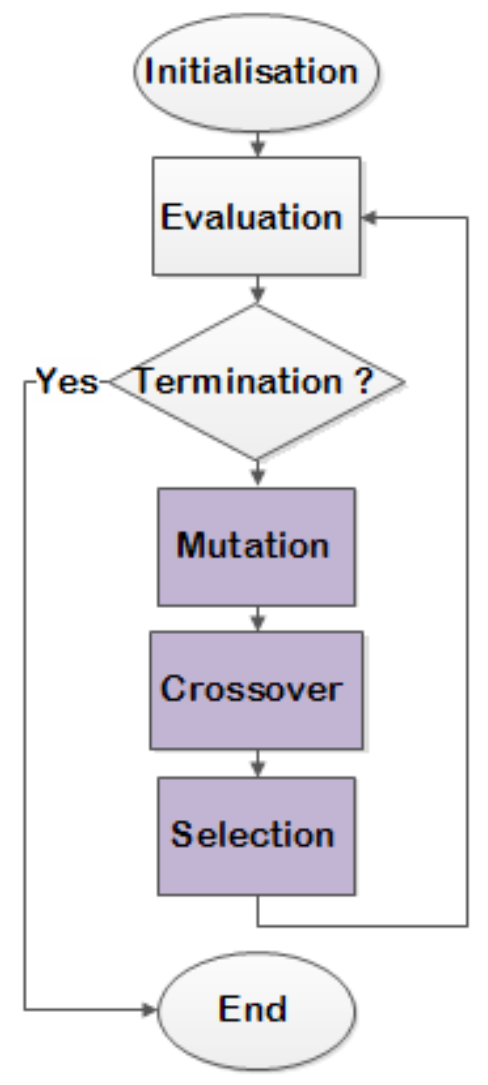

Fig.1. Flowchart of differential evolution algorithm

\section{B. Objective function}

The optimization problem is to minimize a function by assigning the variables on which it depends. More formally, let $E$ be a set of candidate solutions, and $F$ is a function $F: E \rightarrow R$, which is called objective function. It affects the various candidates from $E$ to measurement solutions in $R$. So the optimization problem is to find the solution that minimizes candidate the function $F$.

Based on the second functional presented in [9], in order to stabilize the switched system, we define the objective function as follows:

$$
f(P)=\operatorname{Max}_{i}\left\{\operatorname{eig}\left(P A_{i}+A_{i}^{T} P\right)\right\}, \forall i=1, . ., N
$$

where eig(.) the function that gives the eigenvalues of a matrix. Finally, we obtain finally one $P$ which satisfy the optimization of the switched linear system and the minimization of $f$ for all iterations.

\section{STABILISATION OF SWITCHED LINEAR SYSTEMS}

Consider the switched linear system:

$$
\dot{x}(t)=A_{\sigma(t)} x(t), \quad A_{\sigma(t)} \in A=\left\{A_{1}, A_{2}, \ldots, A_{N}\right\}
$$

Where $x(t) \in \mathbb{R}^{n}$ is the state vector, $A$ is a set of $N$ Hurwitz matrices in $\mathbb{R}^{n \times n}$ and $\sigma(t)$ is an arbitrary signal that define commutation among elements in $A$.

We define a quadratic Lyapunov function candidate as

$V(x)=x^{T} P x, P>0, \quad P \in R^{n \times n}$

Which is positive definite, whose time derivative along any non-zero system trajectory of (4) is required to be negative definite, i.e.

$$
\dot{V}(x)=x^{T}\left(P A_{i}+A_{i}^{T} P\right) x<0, \forall x \neq 0, \forall i \in\{1, \ldots, N\}
$$

For which it is necessary that

$$
P A_{i}+A_{i}^{T} P<0, \quad \forall i \in\{1, \ldots, N\}
$$

Then, if a matrix $P>0$ satisfying (2) and (4) $\forall A_{i} \in A$ exists, the function $V(x)$ is a CQLF for all subsystems

$$
\dot{x}(t)=A_{i} x(t), \quad \forall i \in\{1, \ldots, N\}
$$

and its existence is a guarantee of uniform asymptotic stability of system (4). [5]

Having discussed differential evolution algorithm, let us now investigate the asymptotic stabilization issue for switched linear systems (4) using this methodology. Here, in this note, only state feedback controllers are considered to stabilize the switched linear control system.

$$
\begin{aligned}
& u(t)=-K_{\sigma} x(t), \quad \forall \sigma \in\{1, \ldots, N\} \\
& \text { with } \\
& K_{\sigma}=B_{\sigma}^{T} P, \quad \forall \sigma \in\{1, \ldots, N\}
\end{aligned}
$$

we get the closed-loop system (10) as follows

$$
\dot{x}(t)=\left(A_{\sigma}-B_{\sigma} K_{\sigma}\right) x(t), \quad \forall \sigma \in\{1, \ldots, N\}
$$

For the system to be stable, it is necessary that the eigenvalues of $A_{\sigma}-B_{\sigma} K_{\sigma}$ are strictly negatives.

In this work, the objective function ensures the instability of the system (4) and looks for the best solution in each iteration by minimizing it.

So that, before starting the selection (or evaluation) we have imposed some conditions for our corrected switched systems 
(11) will be stable as the eigenvalues of $P$ must be strictly positives and all the eigenvalues of $\left(A_{\sigma}-B_{\sigma} K_{\sigma}\right)$ are strictly negatives. A reverse method was used.

\section{NUMERICAL EXAMPLES}

To illustrate the main results, two examples are considered.

\section{Example 1:}

Consider the linear switched system defined by

$$
\dot{x}(t)=A_{i} x(t), \quad i \in\{1,2,3\}
$$

where

$$
\begin{aligned}
A_{1} & =\left[\begin{array}{cccc}
-0.4872 & 0 & 0.0123 & -0.0048 \\
0 & 0.4903 & 0 & 0.0274 \\
0.0117 & -0.0035 & 0.8251 & 0.0123 \\
0.0082 & -0.0028 & 0 & 1.2125
\end{array}\right] \\
A_{2} & =\left[\begin{array}{cccc}
1.2246 & 0.0026 & 0 & 0.0163 \\
0.0072 & -0.9181 & 0.0053 & 0.0035 \\
0 & 0.001 & 0.4594 & 0 \\
0.0054 & -0.0048 & 0.0032 & 0.8867
\end{array}\right] \\
A_{3} & =\left[\begin{array}{cccc}
-1.4414 & 0 & -0.0024 & 0.0021 \\
-0.0031 & 0.7321 & 0 & 0.0098 \\
0.0054 & 0 & 0.8534 & 0 \\
0.0051 & -0.0013 & 0.0084 & -0.5621
\end{array}\right]
\end{aligned}
$$

It's evident that the system is initially unstable.

By applying the algorithm of the differential evolution, we obtain the matrix $P$ which is symmetric and positive, defined as:

$$
P=\left[\begin{array}{rrrr}
2.4200 & 0.0057 & 1.6312 & 0.4978 \\
0.0057 & 3.4539 & 0.8349 & -1.2821 \\
1.6312 & 0.8349 & 4.5236 & -0.5129 \\
0.4978 & -1.2821 & -0.5129 & 2.9555
\end{array}\right]
$$

The following figure shows the diagram of all the objective functions obtained in each iteration from 1 to 15000 .

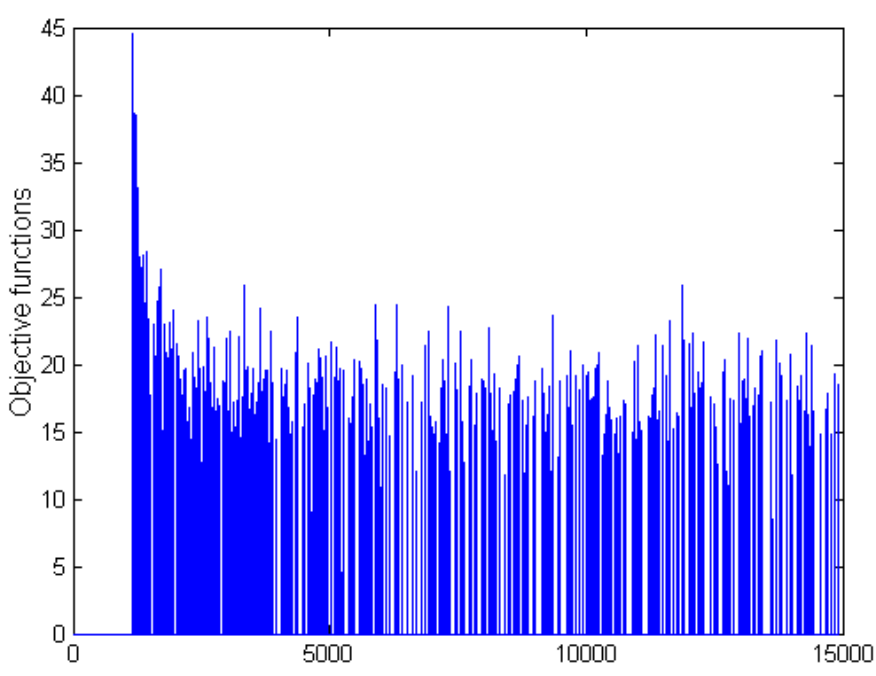

Fig. 2. Values of all objective functions for 15000 iterations

Now, it is very interesting to see just the evolution of the best objective functions during all the iterations.

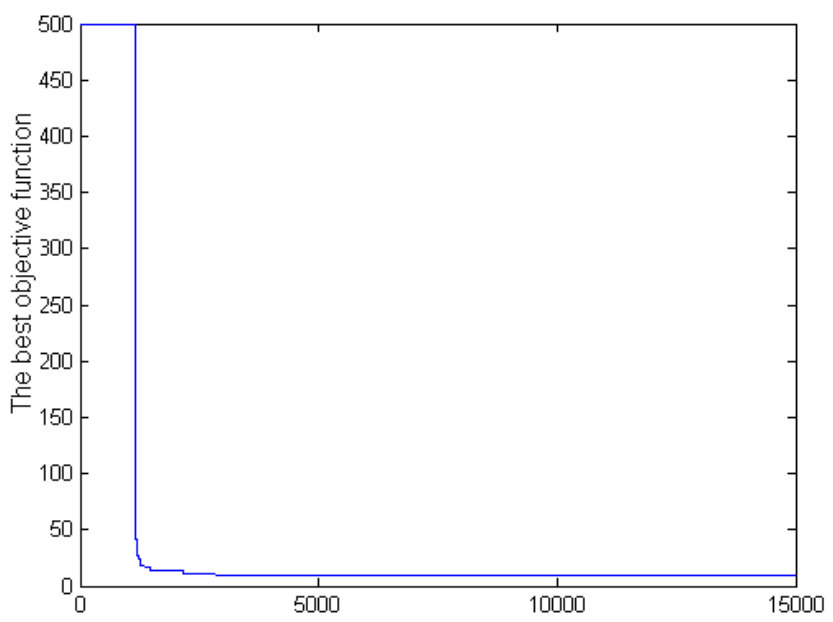

Fig. 3. The evolution of the best objective function for 15000 iterations

It is clear that this is a minimization from the initial value until the optimal solution.

The trajectories of the system (12) after stabilization are given in figures 4.a, 4.b, 4.c and 4.d for a switching during $\mathrm{k}=0,1,2, \ldots, 10$. The switching sequence is shown in figure 4.e.

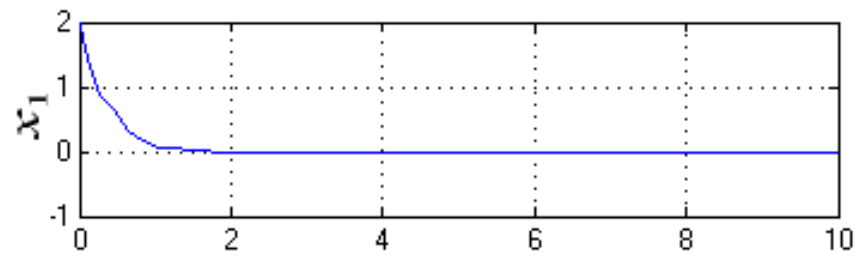

Fig. 4.a. The trajectory of $x_{1}$ 


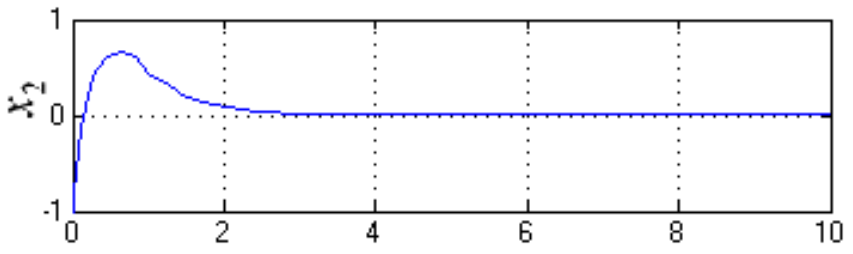

Fig. 4.b. The trajectory of $x_{2}$.

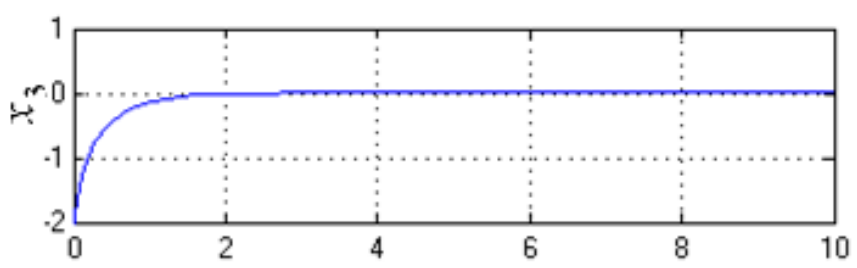

Fig. 4.c. The trajectory of $x_{3}$

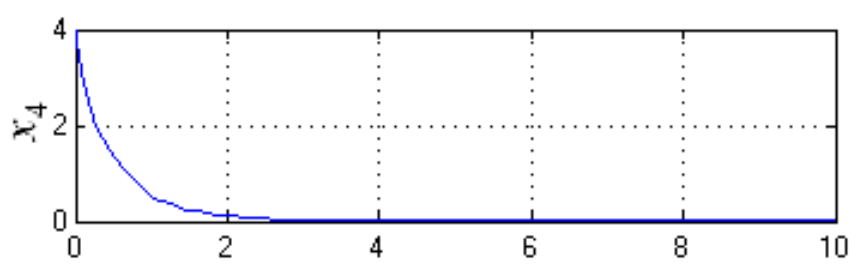

Fig. 4.d. The trajectory of $x_{4}$

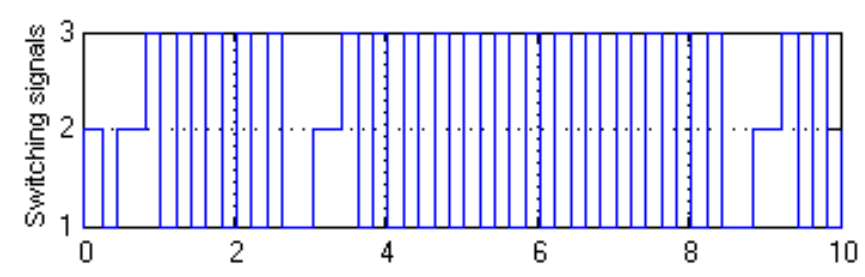

Fig. 4.e. The switching signals

\section{Example 2:}

Consider the linear switched system defined by

$\dot{x}(t)=A_{i} x(t), \quad i \in\{1,2\}$

where

$$
\begin{aligned}
& A_{1}=\left[\begin{array}{cc}
-0.2703 & 0.1266 \\
0 & 1.2214
\end{array}\right] \\
& A_{2}=\left[\begin{array}{ll}
1.2067 & 0.0876 \\
0.2662 & 0.1594
\end{array}\right]
\end{aligned}
$$

After application of the differential evolution algorithm to the switched system (15) and simulation, we obtain the symmetric matrix $P>0$ which satisfies the linear matrix inequality (7) as follow

$$
P=\left[\begin{array}{cc}
1.3282 & -0.2063 \\
-0.2063 & 1.8063
\end{array}\right]
$$

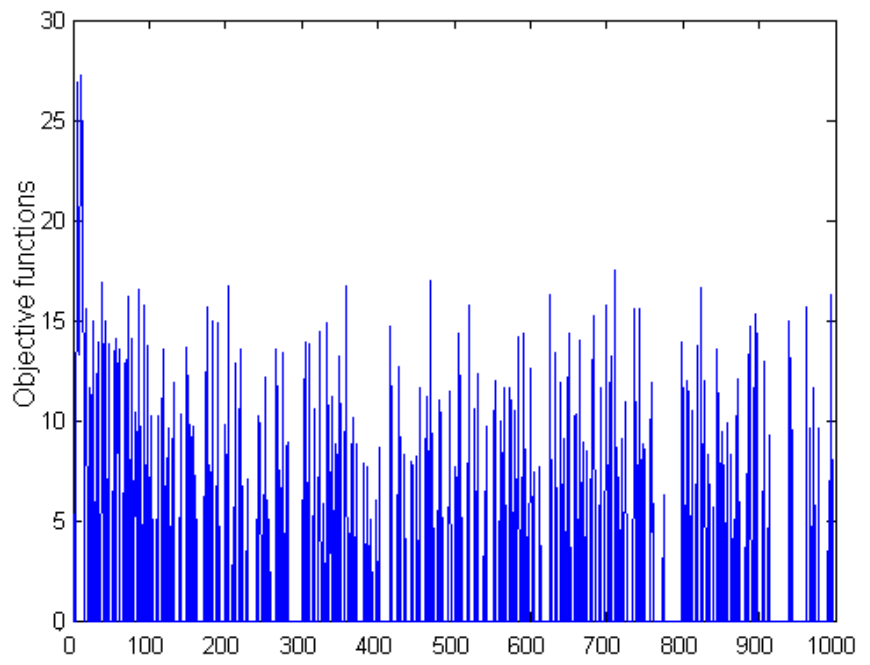

Fig. 5. Values of all objective functions for 1000 iterations

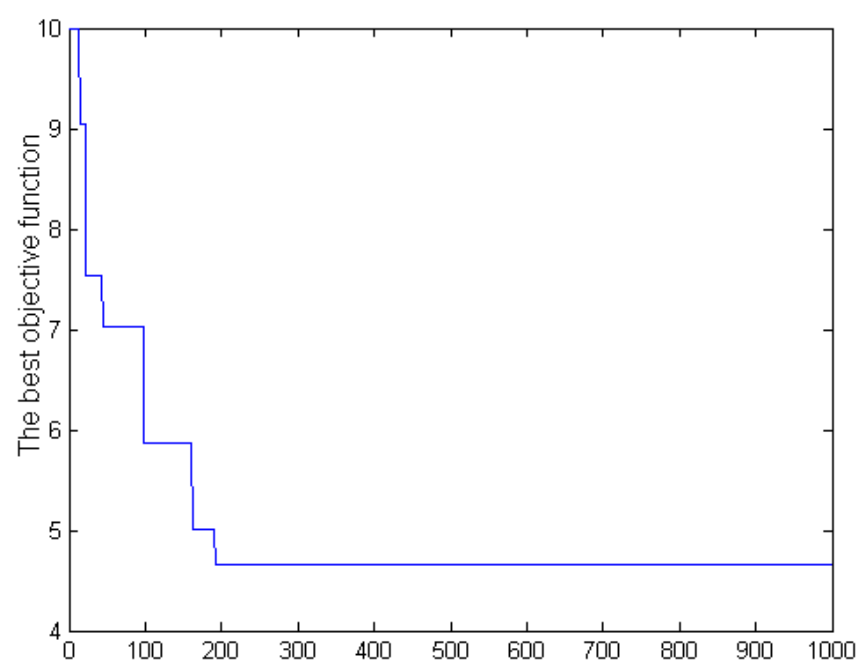

Fig. 6. The evolution of the best objective function for 1000 iterations

The trajectories of the system (15) after stabilization are given in figures 7.a, and 7.b, for a switching during $\mathrm{k}=0,1,2, \ldots, 10$. The switching sequence is shown in figure 7.c.

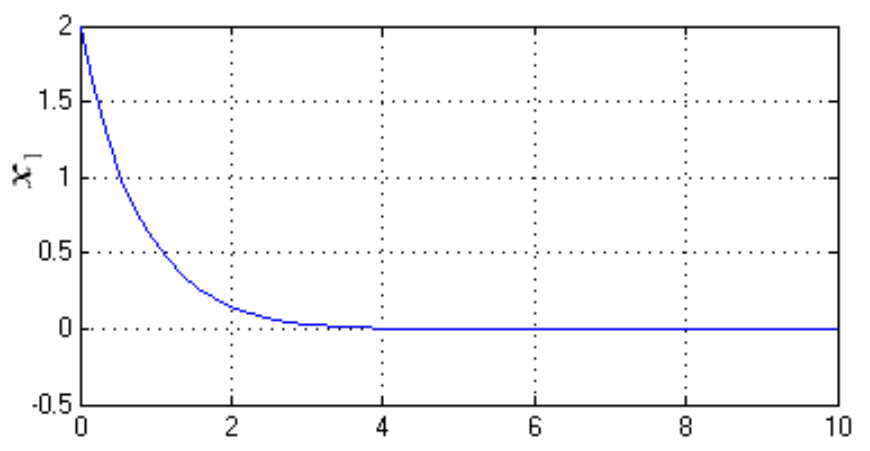

Fig. 7.a. The trajectory of $x_{1}$ 


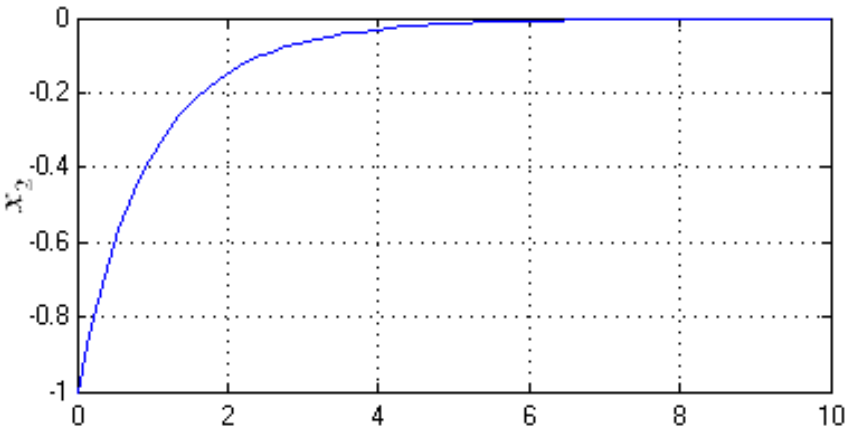

Fig. 7.b. The trajectory of $x_{2}$

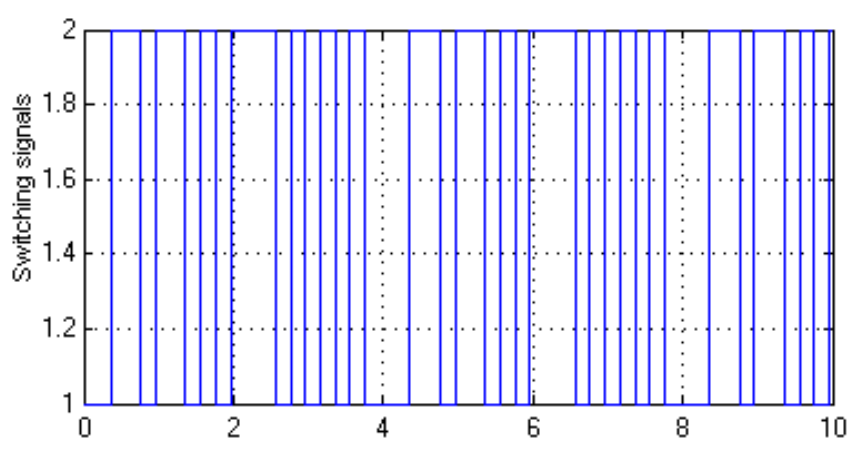

Fig. 7.c. The switching signals

\section{Conclusion}

In this paper a new methodology for stabilization of switched linear systems using the differential evolution algorithm is presented. Two examples is given to illustrate the usefulness of this result. One of the main conclusions reached is that differential evolution algorithm is a successful technique for finding a CQLF. Moreover, differential evolution algorithm is a multiobjective evolutionary algorithm so that we can use it to improve system performances.

\section{References}

1. Agrachev AA, Liberzon D. Lie-algebraic stability criteria for switched systems. SIAM J Control Opt; 41:253-69 (2001).

2. Cheng D, Guo L, Huan J. On quadratic Lyapunov functions. IEEE Transaction Automatic Control; 48:885-90 (2003).

3. Dayawansa WP, Martin CF. A converse Lyapunov theorem for a class of dynamical systems which undergo switching. IEEE Transaction Automatic Control; 44:751-60 (1999).

4. Daafouz J, Riedinger P, Iung C. Stability analysis and control synthesis for switched systems: a switched Lyapunov function approach. IEEE Transaction Automatic Control; 47:1883-7 (1999).

5. Lin, H., and Antsaklis, P.J., 'Stability and Stabilisability of Switched Linear Systems: A Survey of Recent Results', IEEE Transactions on Automatic Control, 54, 308-322 (2009).
6. Rodrigo H. Ordonez-Hurtado and Manuel A. DuarteMermoud, A Methodology for Determining the NonExistence of Common Quadratic Lyapunov Functions for Pairs of Stable Systems, Fifth International Conference on Genetic and Evolutionary Computing, (2011)

7. Juing-Shian Chiou and Chun-Ming Cheng, "Stabilization analysis of the switched discrete-time systems using Lyapunov stability theorem and genetic algorithm" (2009).

8. R.H. Ordóñez-Hurtado and M.A. Duarte-Mermoud, "Finding common quadratic Lyapunov functions for switched linear systems using particle swarm optimization", International Journal of Control, (2012).

9. M.A. Duarte-Mermoud, R.H. Ordóñez-Hurtado and P. Zagalak, "A method for determining the nonexistence of a common quadratic Lyapunov function for switched linear systems based on particle swarm optimization", International Journal of Systems Science,(2012).

10. Liberzon, D., and Tempo, R., 'Common Lyapunov Functions and Gradient Algorithms', IEEE Transactionson Automatic Control, 49, 990994.(2004).

11. Storn, R.M., Price, K.V.: Differential evolution - a simple and efficient adaptive scheme for global optimization over continuous spaces, Technical Report TR- 95-012, ICSI (March 1995).

12. K. Price, R. Storn, and J. Lampinen, Differential Evolution: A Practical Approach to Global Optimization, ser. Natural Computing Series. Springer-Verlag, ( 2005).

13. Zaharie, D.: Control of population diversity and adaptation in differential evolution algorithms. In: Matousek, R., Osmera, P. (eds.) 9th International Conference on Soft Computing, MENDEL (2003).

14. Xue, F., Sanderson, A.C., Graves, R.J.: Modeling and convergence analysis of a continuous multiobjective differential evolution algorithm. In: Proc. of IEEE Congress on Evolutionary Computation, September 2005, vol. 1, pp. 228-235 (2005).

15. Xue, F.: Multi-Objective Differential Evolution: Theory and Applications. Ph.D. dissertation, Rensselaer Polytechnic Institute, Ney York, USA (2004).

16. Swagatam Das, Ponnuthurai Nagaratnam Suganthan, Differential Evolution: A Survey of the State-of-theArt. IEEE Transactions on evolutionary computation, vol. 15, No. 1, (February 2011). 